\title{
Subwavelength localization and toroidal dipole moment of spoof surface plasmon polaritons
}

\author{
Seong-Han Kim,,${ }^{1,2, *}$ Sang Soon Oh, ${ }^{3}$ Kap-Joong Kim, ${ }^{4}$ Jae-Eun Kim, ${ }^{5}$ Hae Yong Park, ${ }^{5}$ Ortwin Hess, ${ }^{3, \dagger}$ and Chul-Sik Kee ${ }^{1,2, \ddagger}$ \\ ${ }^{1}$ Integrated Optics Laboratory, Advanced Photonics Research Institute, GIST, Gwangju 500-712, South Korea \\ ${ }^{2}$ Center for Subwavelength Optics, Seoul 151-742, South Korea \\ ${ }^{3}$ Blackett Laboratory, Department of Physics, Imperial College London, London SW7 2AZ, United Kingdom \\ ${ }^{4}$ Electronics and Telecommunications Research Institute, Daejeon 305-700, South Korea \\ ${ }^{5}$ Department of Physics, Korea Advanced Institute of Science and Technology, Daejeon 305-701, South Korea
}

(Received 5 November 2014; published 13 January 2015)

\begin{abstract}
We experimentally and theoretically demonstrate subwavelength scale localization of spoof surface plasmon polaritons at a point defect in a two-dimensional groove metal array. An analytical expression for dispersion relation of spoof surface plasmon polaritons substantiates the existence of a band gap where a defect mode can be introduced. A waveguide coupling method allows us to excite localized spoof surface plasmon polariton modes and measure their resonance frequencies. Numerical calculations confirm that localized modes can have a very small modal volume and a high $Q$ factor both of which are essential in enhancing light-matter interactions. Interestingly, we find that the localized spoof surface plasmon polariton has a significant toroidal dipole moment, which is responsible for the high $Q$ factor, as well as an electric quadrupole moment. In addition, the dispersion properties of spoof surface plasmon polaritons are analyzed using a modal expansion method and numerical calculations.
\end{abstract}

DOI: 10.1103/PhysRevB.91.035116

PACS number(s): 78.67.Pt, 41.20.Jb, 42.50.Pq, 73.20.Mf

\section{INTRODUCTION}

Ultrasmall cavities with a high quality factor ( $Q$ factor) are vital elements in modern photonics. They are widely used not just to miniaturize photonic devices but also to enhance light-matter interactions such as spontaneous emission [1,2] and nonlinear optical processes [2-4]. In particular, the ability of controlling spontaneous emission of emitters is a core element in the fields of both cavity quantum electrodynamics (QED) that employs real atoms [4-7] and circuit QED that involves Josephson junctions (JJs) [8-13]. Accordingly, up to now, many researchers have suggested various types of cavities to achieve an ultrasmall modal volume and a high $Q$ factor, for example, dielectric or plasmonic cavities for single-photon sources, and microwave stripline cavities for microwave circuit QED. In the optical region, photonic crystal (PC) cavities [14], a type of dielectric cavity, have been employed to confine electromagnetic (EM) waves at a defect site for a band gap frequency range allowing its modal volume to approach the theoretical diffraction limit, a cubic half wavelength in the dielectric medium [15-17]. To realize a cavity with an even smaller size beyond the diffraction limit, plasmonic cavity systems have recently been employed. It has been demonstrated that a plasmonic cavity system, based on surface plasmon polaritons (SPPs) [18] propagating along a metal-dielectric interface, can confine EM waves at the deep subwavelength scale and enhance EM fields using various geometries, such as particles [19-21], rings [22,23], and holes [24-27]. In plasmonics, it was suggested that even stopped light lasing could be realized with a high spontaneous emission factor $\beta$ [28]. In contrast, in lower frequency regions such as terahertz and microwave, less attention has been paid to plasmonic cavity systems due to weak field confinement near a metal-dielectric

\footnotetext{
*topofuni@gmail.com

†o.hess@imperial.ac.uk

†cskee@gist.ac.kr
}

surface. The weak field confinement is because metals behave like a perfect electric conductor (PEC) in the frequency regions preventing a tightly bound EM surface mode. Therefore, it is a challenging task to achieve strong field localization and field enhancement for realizing an ultrasmall cavity with a high $Q$ factor in the terahertz and microwave regions.

To overcome the limitation of field confinement in the lower frequency regions, a concept of "spoof surface plasmon polaritons," which can be excited in a periodically structured PEC surface, has been proposed [29]. The periodically structured PEC surface behaves effectively like a planar metal surface at optical frequencies showing tightly bound EM modes for lower frequencies and, more importantly, propagation properties of spoof SPPs can be controlled by engineering the geometric parameters [30-34]. Therefore, spoof SPPs can be manipulated to imitate the EM behavior of SPPs such as guiding [34-40], localization [39,41-43], and focusing $[33,37,44,45]$. Remarkably, some structured metal surfaces exhibit a spoof SPP band gap, where no surface guided modes are allowed. An example of such a structure is a $2 \mathrm{D}$ groove metal array (GMA), i.e., two perpendicular and overlapping $1 \mathrm{D}$ groove metal arrays which can be regarded as a two-dimensional array of square metal rods on a flat metal surface. It has been reported that a 2D GMA can support additional higher order surface bound modes as well as the lowest spoof SPP modes with numerical [46] and experimental [47] demonstrations of their propagation properties. Moreover, one can find out a band gap between the surface bound states in the 2D GMA [46,47]. It is worth noting that the spoof SPP as a polariton is a result of strong coupling of EM waves with geometrically induced EM excitations and a band gap arises in its dispersion, due to the anticrossing principle. The polaritonic band gap with geometrically controllable properties could allow us to localize EM radiations in a small defect site such as a photonic band gap cavity.

On the other hand, it has been demonstrated that a toroidal dipole resonance, which has received little attention for many 
years, is an important EM response in certain metamaterials, called "toroidal metamaterials." Toroidal metamaterials [4854] have been designed to maximize the toroidal dipole response, which is formed by a poloidal current on a torus surface and cannot be described in a standard multipole expansion $[55,56]$ unlike electric multipoles and magnetic multipoles. Due to the unique features of toroidal dipoles such as strong EM energy confinement and weak coupling to free space [57], toroidal metamaterials are expected to have potential application in enhancing light-matter interactions. This suggests that a higher $Q$ factor could be achieved if a toroidal geometry is employed to maximize the toroidal dipole response in cavity applications.

In this work, we derive the dispersion relation of surface modes in a 2D GMA and then identify the origin of the spoof SPP band gap, which differs from that of a PC band gap. Although the origin of the spoof SPP band gap is different from that of a PC band gap, we show that localized spoof SPPs can be created by introducing a point defect mode, which exhibits a toroidal dipole moment, in a similar manner to 2D PC cavities. We experimentally verify the existence of the band gap and the defect modes by measuring the transmission spectrum and using a waveguide coupling method, respectively. From numerical calculation results for the resonant modes in the defect structures, we investigate the mode properties such as modal volume, $Q$ factor, and toroidal dipole moment.

\section{DISPERSION RELATION OF TWO-DIMENSIONAL GROOVE METAL ARRAY}

The 2D GMA investigated in this study is composed of two overlapping 1D groove metal arrays perpendicular to each other with lattice constant $a$, width $w$, and depth $h$ as shown in Fig. 1(b). We derive the dispersion relation of TM-polarized surface modes in the 2D GMA by using a modal expansion method of EM fields. Due to the square symmetry of our structure, we can, without loss of generality, consider only the modes propagating along the $x$ direction (i.e., $k_{y}=0$ ). For simplicity, we first disregard the time dependence of the EM fields, $\exp (-i \omega t)$, where $\omega$ is the angular frequency of the modes. First, since the width of grooves is much smaller than the wavelength, only TEM modes are considered in the

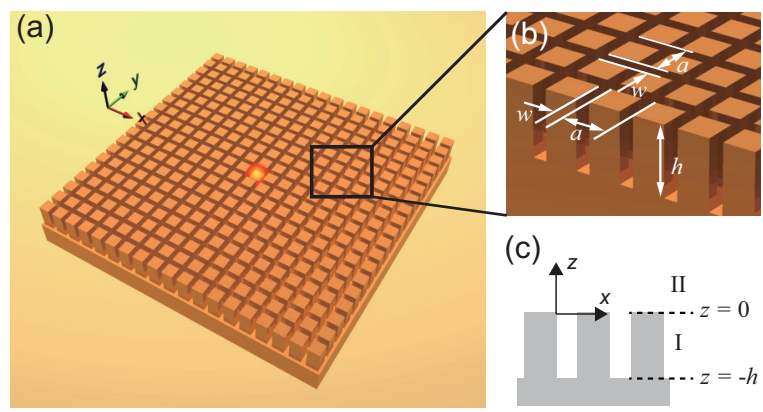

FIG. 1. (Color online) (a) An illustration of localized spoof SPPs at a point defect in 2D GMA formed on a metallic surface. (b) Schematic diagram of the 2D GMA with groove depth $h$, width $w$, and periodicity $a$. (c) Schematic diagram for deriving dispersion relations of TM modes. expansion of EM fields in the grooves (region I):

$$
H_{y}^{\mathrm{I}}=A^{+} \exp \left(i k_{0} z\right)+A^{-} \exp \left(-i k_{0} z\right),
$$

where $A^{+}$and $A^{-}$are the amplitudes of the magnetic fields, $k_{0}(=\omega / c)$ is the wave number in vacuum with $c$ the speed of light in vacuum, and the + and - signs of the exponents correspond to $+z$ and $-z$ directions, respectively. Second, the magnetic field component $H_{y}$ outside the GMA (region II) can be expressed as

$$
H_{y}^{\mathrm{II}}=\sum_{n=-\infty}^{\infty} B_{n} \exp \left[-\alpha_{n} z+i k_{x, n} x\right],
$$

where $B_{n}$ 's are constants, $k_{x, n}=k_{x}+2 \pi n / a$ is the $x$ component of the wave vector of the $n$th diffraction order, and $\alpha_{n}=\sqrt{k_{x, n}^{2}-k_{0}^{2}}$. The other nonzero components of the EM fields can be determined directly from $H_{y}^{\mathrm{II}}$. It is worth noting that $k_{x}$ must be outside the light cone so that the EM fields are confined at the surface. The dispersion relation of the surface modes is obtained by applying continuous boundary conditions on $H_{y}$ and $E_{x}$ at the air-groove interface and at the bottom of the grooves, respectively. By following this step, we obtain a transcendental equation:

$$
\cot \left(k_{0} h\right)=\sum_{n=-\infty}^{\infty} \frac{k_{0} S_{n}^{+} S_{n}^{-}}{\sqrt{k_{x, n}^{2}-k_{0}^{2}}},
$$

where $\quad S_{n}^{+}=\sqrt{w / a}\left\{\operatorname{sinc}\left(k_{x, n} w / 2\right)+\operatorname{sinc}\left(k_{x, n} a / 2\right)-(1-\right.$ $\left.w / a) \operatorname{sinc}\left[k_{x, n}(a-w) / 2\right]\right\}$ and $S_{n}^{-}=\sqrt{w / a} \operatorname{sinc}\left(k_{x, n} w / 2\right)$. In addition to the TM-polarized surface modes, there exist TE-like hybrid surface modes in our structure. In Appendix A, we derive dispersion relations for these modes under a perfect magnetic conducting wall assumption.

Figures 2(a) and 2(b) show the dispersion relations of surface modes propagating along the $x$ direction in the 2D GMA with $h=a$ and $h=3 a$ obtained by using Eq. (3) and Eq. (A4) for TM modes and TE-like hybrid modes, respectively. In the calculation, we include only the first diffraction order in the summation because sufficient convergence is achieved. For the case of $h=a$, we examine the width dependence by increasing the width from $0.1 a$ to $0.5 a$ and, for the case of $h=3 a$, we analyze higher order modes with groove width kept to be $0.4 a$. In Figs. 2(a) and 2(b), one can clearly see that the dispersion relations are strongly affected by groove depth $h$. To validate our model, we calculated band structures for unit cell structures using the finite element method (FEM). In Fig. 2(b), the guided bands obtained by the numerical calculation for the cases of $h=3 a$ and $w=0.4 a$ are plotted. One can see a good agreement between the bands obtained from analytical dispersion equations and the FEM result.

In the limit of $a \ll \lambda$, we can safely neglect the non-zerothorder diffraction terms in Eq. (3):

$$
\frac{\sqrt{k_{x}^{2}-k_{0}^{2}}}{k_{0}}=S_{0}^{+} S_{0}^{-} \tan \left(k_{0} h\right) .
$$

It is worth noting that, in the long wavelength limit $\left(k_{x} a \ll 1\right)$, $S_{0}^{+} S_{0}^{-}$reduces to $S_{0}^{+} S_{0}^{-} \simeq w / a+(w / a)^{2}$. Moreover, for a very small groove width $(w \ll a), S_{0}^{+} S_{0}^{-}$can be approximated by $w / a$, so that the Eq. (4) coincides with Eq. (14) in Ref. [30]. 


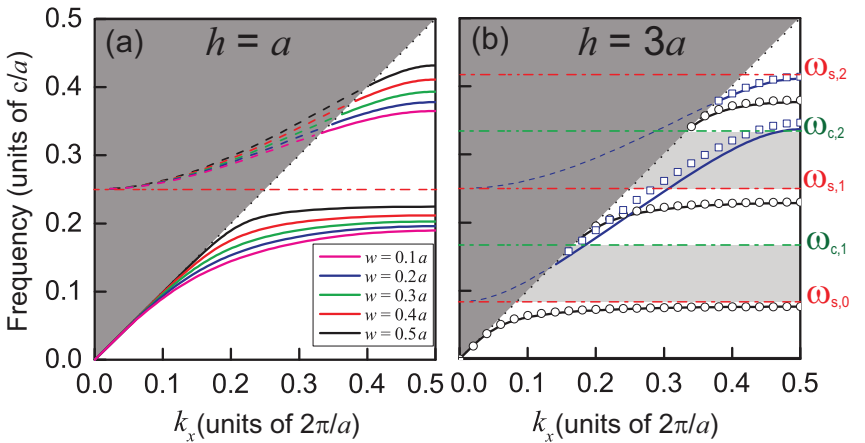

(c)
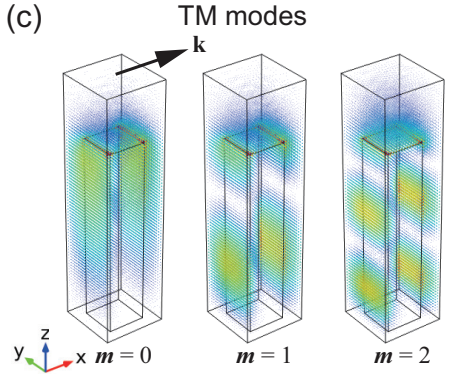

(d)

d) TE-like modes

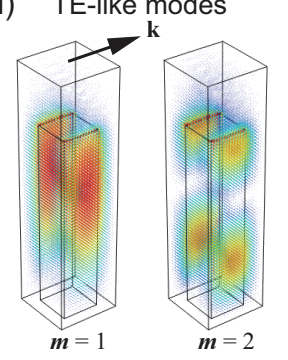

FIG. 2. (Color online) (a) Dispersion relations (solid lines) of surface modes propagating along the $x$ direction for the 2D GMA with $h=a$ calculated for increasing values of gap width $w$. The dash-dotted line is the asymptotic frequency, $\omega_{s, 0}$. The modes below $\omega_{s, 0}$ are TM-polarized and the modes above $\omega_{s, 0}$ are TE-like hybrid modes. Dashed lines denote radiation modes of TE-like hybrid bands. (b) Dispersion relations of TM modes (black solid lines) $m=0,1$, and 2, and TE-like hybrid modes (blue solid lines) $m=1$ and 2 for the structure with $h=3 a$ and $w=0.4 a$. The open dots denote the bands from FEM calculations for TM modes (circle) and TE-like hybrid modes (square). Dash-dotted lines stand for asymptotic frequencies (red) and cutoff frequencies (green) of TM modes. Dark shaded regions and lightly shaded regions denote radiation modes and TM band gaps, respectively. (c), (d) Simulated amplitudes of the electric field in a unit cell for TM modes $(m=0,1,2)$ and TE-like hybrid modes $(m=1,2)$ at $k_{x}=\pi / a$ in (b). Black arrows indicate the propagation direction.

This means that a 2D GMA can be regarded as an effective medium that supports surface modes known as spoof SPPs. In addition, as pointed out in Ref. [46], there is a close similarity in the field distributions and dispersion relations between the 2D GMA and the 1D GMA. In Fig. 2, the dispersion curve of the fundamental TM mode $(m=0)$ is very similar to that of SPPs at a metal-dielectric interface in the visible or nearinfrared frequency region. In both cases, the dispersion curves exhibit asymptotic behaviors for large wave vectors $k_{x}$. In the case of SPPs, $\omega$ approaches $\omega_{p} / \sqrt{2}$, where $\omega_{p}$ is the plasma frequency of metals, whereas, in this case, Figs. 2(a) and 2(b) show that $\omega$ approaches $\omega_{s, 0}(=\pi c / 2 h)$. Also, note that the asymptotic frequency is mainly controlled by the depth of the grooves, as shown in Fig. 2.

Interestingly, if the groove depth is deep enough (i.e., $h>a$ ), the dispersion equation [Eq. (3)] of spoof SPP allows for higher order modes in the subwavelength regime as shown in Fig. 2(b) (which is a main different feature to dispersion properties of conventional SPPs). In general, the frequency of surface modes in a 2D GMA has lower and upper limits showing a frequency band with a finite bandwidth. The lower limit is the cutoff value $\omega=c k_{x}$, at which the surface mode extends to $z=\infty$. According to the mode condition $\left[\tan \left(k_{0} h\right)=0\right]$, cutoff values of $\omega$ for TM surface modes are given as $\omega_{c, m}=m \pi h / c$, where $m$ is an integer $(m=0,1,2,3, \ldots)$ which refers to the $(m+1)$ th band. And, the upper limit is the asymptotic frequency which is given as $\omega_{s, m}=(m+1 / 2) \pi h / c$. Thus, the TM surface waves are allowed to propagate only with the frequency between the two limits. In addition, for the band in Fig. 2(b), we calculate the electric field distributions of the three TM modes ( $m=0,1,2)$ and two TE-like hybrid modes $(m=1,2)$ at the $X$ point $\left(k_{x}=\pi / a\right)$ of the first Brillouin zone, as depicted in Figs. 2(c) and 2(d), respectively. Interestingly, the asymptotic frequency for the $(m+1)$ th band is the $(m+1)$ th resonance frequency of the individual cavity which can be regarded as a transmission line cavity [58]. This confirms that the TM surface modes derive from a coupling between free photons and resonant modes in the individual transmission line cavity.

\section{SPOOF SPP BAND GAP}

As shown in Fig. 2(b), band gaps can exist for spoof SPP modes in a 2D GMA and they have several interesting characteristics. First of all, the origin of spoof SPP band gaps is not a Bragg reflection as for the case of PC band gaps, but is associated with the polaritonic nature of spoof SPPs. As mentioned previously, the spoof SPPs in a 2D GMA originate from the coupling between free photons and resonant modes in the transmission line cavities. The coupling creates the spoof SPP band gaps between the upper edge of the $(m+1)$ th TM mode and the cutoff frequency of the $(m+2)$ th TM modes [see the shaded regions in Fig. 2(b)]. This coupling is similar to the case of ionic crystals where phonon polaritons originate from the coupling between photons and transverse optical phonons and exhibit a band gap between the transverse optical phonon frequency and longitudinal phonon frequency [59]. Second, the spoof SPP band gap is not a complete band gap because there are still radiation modes at those frequencies in contrast to band gaps of ionic crystals. It is noteworthy that the incomplete band gap does not forbid defect modes at the band gap frequencies and they can still allow defect modes such as a cavity mode and a waveguide mode as in the cases of two-dimensional PC slabs [60]. Lastly, the spoof SPP band gap is only for TM polarization. Therefore, different polarization modes can be supported at the band gap frequencies. Figures 2(a) and 2(b) show that the first-order TE-like hybrid mode $(m=1)$ partially overlaps with the first band gap of the TM mode. The cutoff frequency of TE-like hybrid modes, at which its band intersects the light line, is, however, higher than the band edge of the fundamental TM mode $(m=0)$. As a result, a band gap region can exist not allowing any guided modes for both polarizations, although the size of the band gap is smaller. Furthermore, we can notice that the 2D GMA always has spoof SPP band gaps even for a very small lattice constant $a$.

To experimentally demonstrate the presence of the band gap, we measured transmission spectrum of surface modes for microwave frequencies. Figure 3(a) shows the sample measurement scheme used to measure the fields of surface modes 


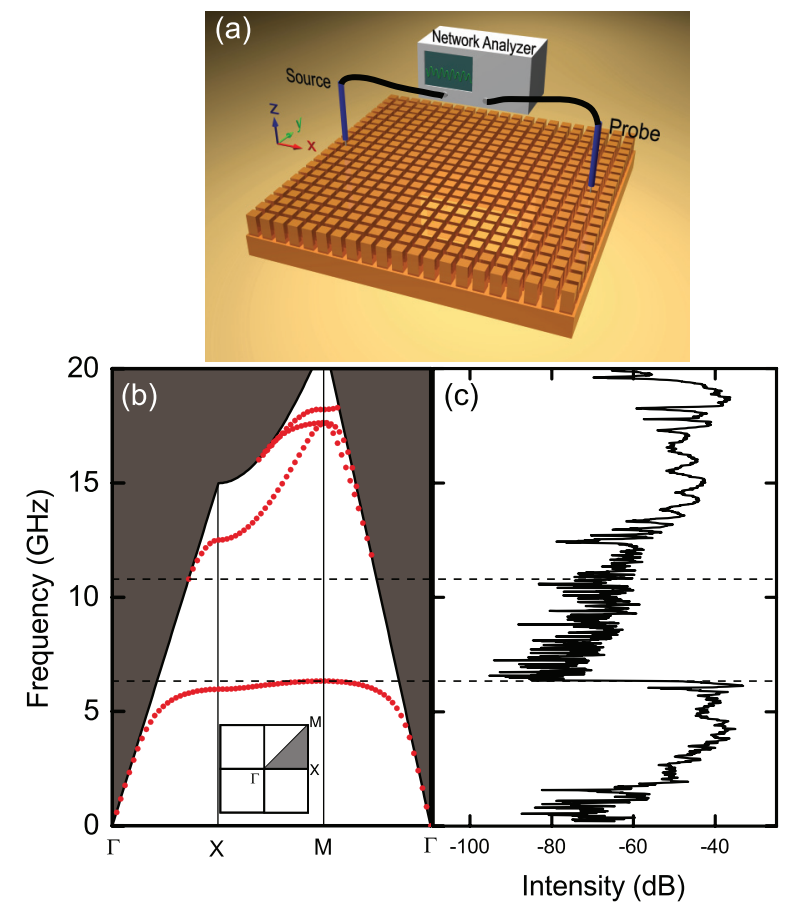

FIG. 3. (Color online) (a) Schematic of experimental setup to measure fields of propagating modes from the source. (b) Projected photonic band structure of surface modes along the boundary of the first irreducible Brillouin zone calculated by FEM for $a=10 \mathrm{~mm}$, $h=10 \mathrm{~mm}$, and $w=4 \mathrm{~mm}$. Radiation modes (shaded region) above the light cone were not displayed. (c) Transmission spectrum for surface modes propagating along the $x$ direction between the two antennas. Note that since the antennas are inefficient, the absolute values of intensity are meaningless.

between two monopole antennas. A pair of small monopole antennas, which are connected to a network analyzer, were used as a source and a detector to measure the electric fields of surface modes $[38,47]$. The two monopole antennas (with dimensions of $1.6 \mathrm{~mm}$ in diameter and $10 \mathrm{~mm}$ in length) are oriented in the direction normal to the surface of the samples.
Our measurements are conducted inside a chamber consisting of a $20 \mathrm{~dB}$ absorber. Using a network analyzer, we measure the $S$ parameter which is proportional to the transmission between the two antennas. When we interpret the measured spectrum from the setup, we compare relative $S$ parameter values since the absolute values of $S$ parameters are meaningless due to very large impedance of the antennas.

To obtain transmission spectra for surface modes using the measurement setup, we fabricate a sample with dimensions of $22 \mathrm{~cm} \times 22 \mathrm{~cm}$ by assembling copper blocks with parameters $h=10 \mathrm{~mm}, w=4 \mathrm{~mm}$, and $a=10 \mathrm{~mm}$. The sample structure has the band diagram depicted in Fig. 3(b), which is obtained from an FEM simulation. The band diagram exhibits a band gap between the first TM and TE-like hybrid modes. Here, we measure the transmission spectrum for the $x$ direction, which is plotted in Fig. 3(c). In the transmission spectrum, the intensity is about $40 \mathrm{~dB}$ in the TM band and sharply drops to $90 \mathrm{~dB}$ in the band gap region and then gradually increases in the TE-like hybrid band. One can clearly confirm the band gap in frequency range from $6.3 \mathrm{GHz}$ to $11 \mathrm{GHz}$ where the transmitted intensities have lower values from -90 $\mathrm{dB}$ to $-60 \mathrm{~dB}$ with noisy signals. Moreover, the transmitted intensity in the band gap exhibits an asymmetric shape, which is evidence of the aforementioned polaritonic band gap.

\section{DEFECT MODE}

Our next step is to introduce a defect mode in the 2D GMA structure. For this purpose, we partially reduce the height of a rod, keeping the rest of the structure unchanged, as shown in the inset of Fig. 4(a). The resonance frequency of the defect mode obtained by FEM simulations increases as the height of the defect rod decreases as shown in Fig. 4(a). Also, the electric field distribution of the defect mode obtained by finitedifference time-domain simulations reveals that a localized spoof polariton mode can be formed at the surface of the 2D GMA. Figures 4(b) and 4(c) show that the spatial distributions of electric and magnetic fields of the defect mode are strongly confined around the defect rod with the height $h_{d}=0.9 a$.
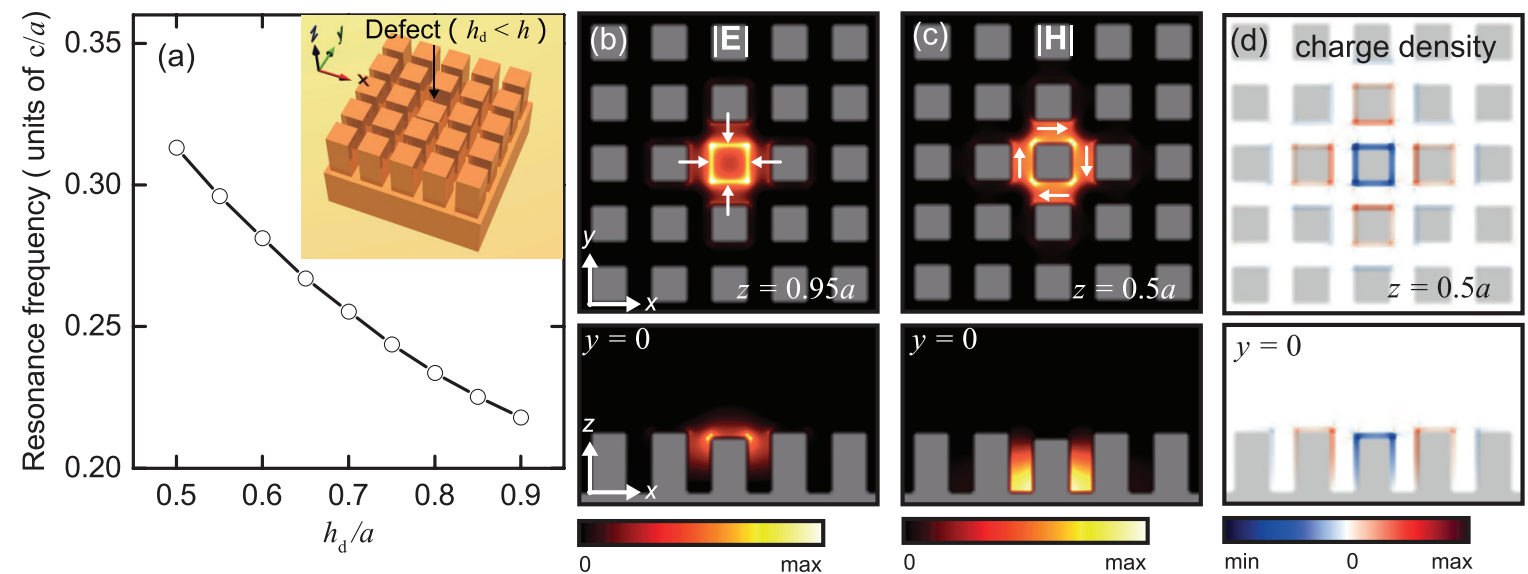

FIG. 4. (Color online) (a) The resonance frequency for increasing values of defect height $h_{d}$ with fixed $h=a$. The inset schematically shows an example of a defect structure, in which the height of one rod is reduced. $h_{d}$ denotes the height of a defect rod. Simulated electric field (b), magnetic field (c), and charge density (d) distributions of point defect modes for the defect structure with $h=a$ and $h_{d}=0.9 a$ at $z=0.95 a$ plane, $z=0.5 a$ plane, and $z=0.5 a$, respectively, and $y=0$ plane. The white arrows denote the directions of the field. 

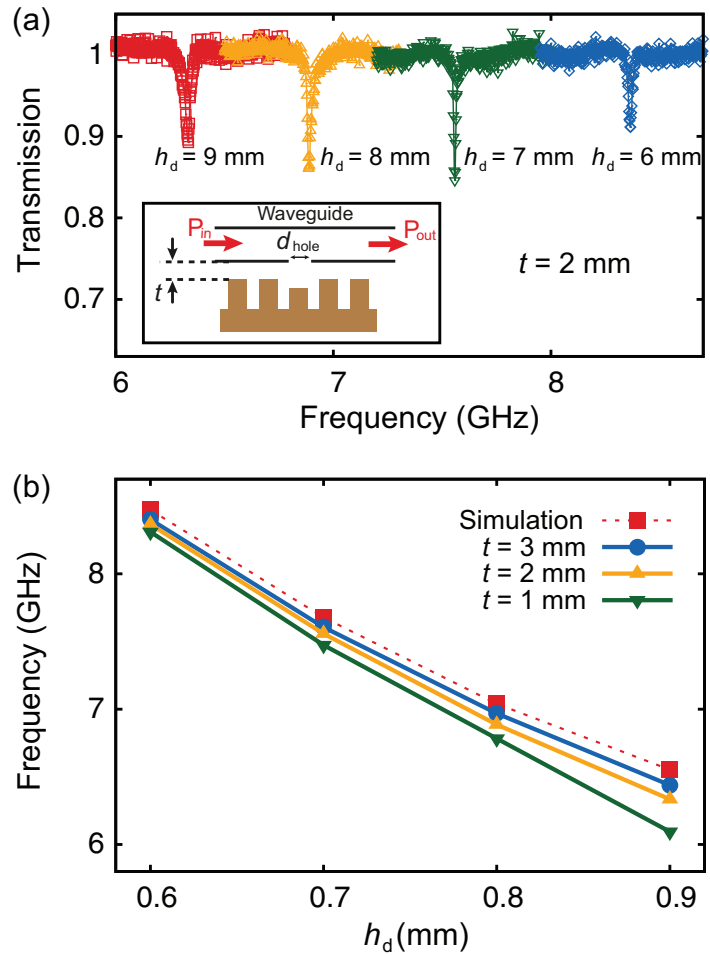

FIG. 5. (Color online) (a) Transmission spectrum at the distance $t=2 \mathrm{~mm}$ for the defect structure for various defect heights with fixed $a=10 \mathrm{~mm}$ and $h=a$. The inset shows a schematic diagram of a waveguide coupling method for excitation of the defect modes. (b) Resonance frequency vs defect height for coupling distances $t=1$, 2 , and $3 \mathrm{~mm}$.

From the distribution of the charge density, shown in Fig. 4(d), we can explain the field distribution of the defect modes.

To verify the localized spoof SPPs in experiment, the waveguide-cavity coupling method [58] was employed as shown in the inset of Fig. 5(a). The resonant coupling of waveguide modes and the defect mode forms a dip at the defect frequency in a transmission spectrum through a waveguide above the sample. Figure 5(a) shows the normalized experimental transmission spectrum through the waveguide for various point defects for $t=2 \mathrm{~mm}$. Figure 5(b) shows a good agreement between experimental and numerical results, except when the distance $t$ between the cavity and waveguide is too close. Such deviation comes from perturbation by the waveguide.

To understand the properties of the observed defect modes, we evaluated their modal volume and $Q$ factors. The modal volume of the defect mode is calculated with the well-known formula

$$
V_{m}=\frac{\int \varepsilon|\mathbf{E}|^{2} d V}{\max \left(\varepsilon|\mathbf{E}|^{2}\right)},
$$

where $\varepsilon$ is the dielectric constant and $\mathbf{E}$ is the electric field [61]. Figure 6(a) shows the calculated modal volumes and $Q$ factors for various defect heights with $h=a$. The modal volume of the defect mode increases as the height of the defect rod decreases, whereas the $Q$ factor decreases as the height of the defect rod decreases. Furthermore, to determine the dependence of the
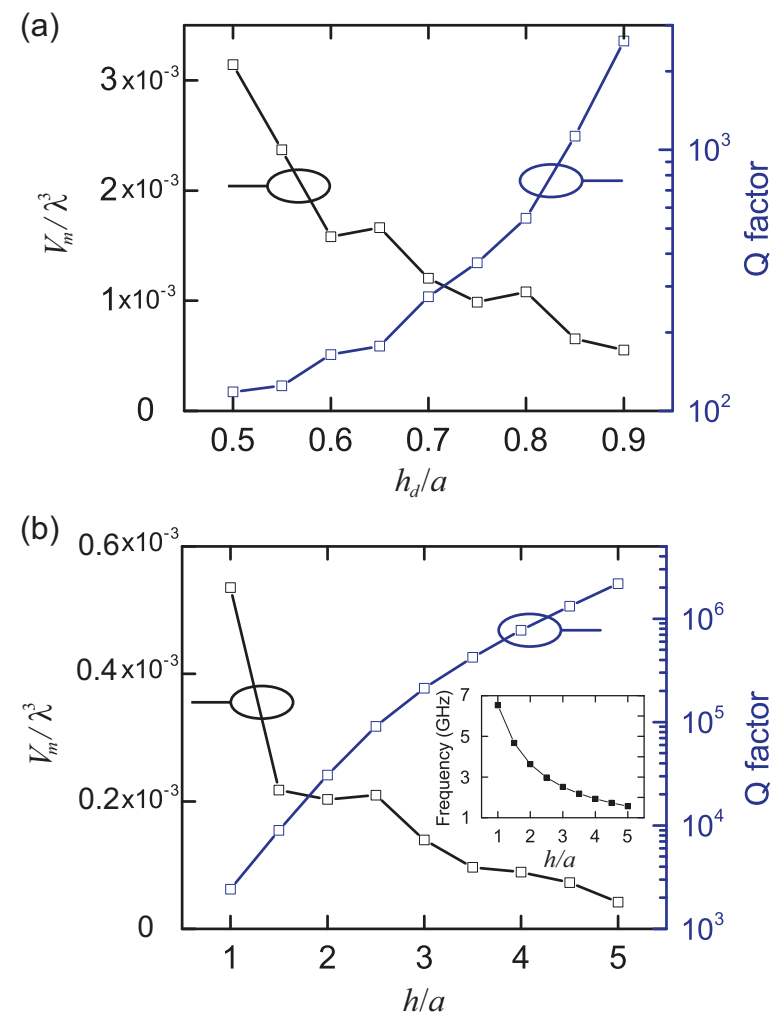

FIG. 6. (Color online) (a) Calculated $Q$ factors and modal volumes of defect modes for increasing values of $h_{d}$ with fixed $a$ and $h=a$. (b) Calculated $Q$ factors and modal volumes of the defect modes for increasing values of groove depths $h$ with fixed $h_{d}=0.9 h$. The inset shows the resonance frequency for the structures.

groove depth $h$ on the modal volume and $Q$ factor of defect modes, we calculated those for the various groove depths with the defect height kept to be $0.9 h$, which are plotted in Fig. 6(b). It would be expected from the dispersion relations and is verified in Fig. 6(b) that the resonance frequency is almost inversely proportional to the groove depth. The modal volume decreases and the $Q$ factor increases as the groove depth $h$ increases, as depicted in Fig. 6(b). In the case of modal volume, it is supposed that some numerical error in the calculation of Eq. (5) is responsible for the unsmooth curve shape. Assuming that a resonant mode is well confined at a defect site, we can estimate that the normalized modal volume is roughly proportional to $a^{2} h / \lambda_{0}^{3} \propto a^{2} / h^{2}$ since $\lambda_{0} \propto h$. Such dependence on the groove depth $h$ provides a possibility of localizing EM fields at a small volume of deep subwavelength scale and further enhancement of the Purcell effect would be possible by simply increasing groove depth, as well.

To obtain deeper physical insight, we investigated the multipole response of defect modes. Considering the geometry of the defect structure, we can expect various multipole responses such as a toroidal dipole moment and an electric quadrupole moment as well as an electric dipole moment. In particular, an induced current distribution, which can be deduced from the charge distributions [Fig. 4(d)], consists of four current loops, i.e., magnetic dipoles around the defect rod reminiscent of a poloidal current in a toroidal coil. Such a poloidal current induces a magnetic toroidal dipole moment 


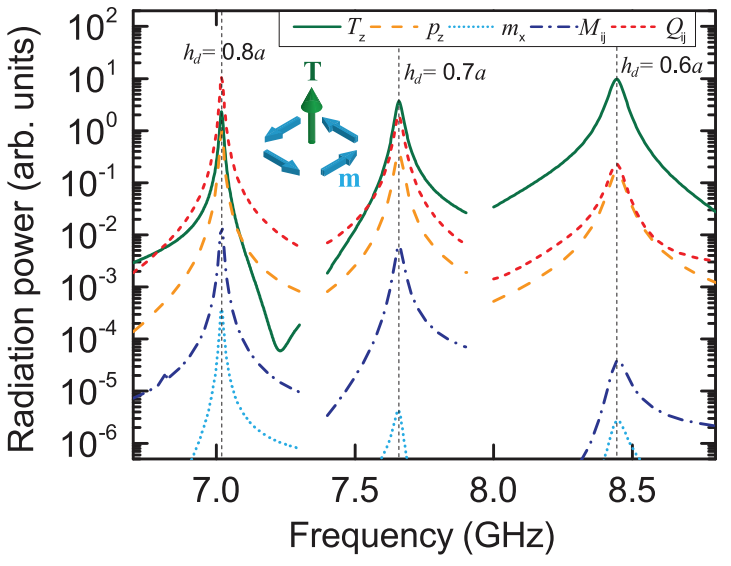

FIG. 7. (Color online) Numerically calculated dispersion of radiating powers of various multipole moments induced for the defects with different defect heights. Dashed vertical lines indicate the resonance positions for the defects with $h_{d}=0.6 a, 0.7 a$, and $0.8 a$. The inset shows an illustration of the generated toroidal dipole moment in this case. Cyan arrows and a green arrow indicate the magnetic dipole moment $(\mathbf{m})$ and the toroidal dipole moment $(\mathbf{T})$.

oriented normal to the corrugated surface, whereas its net magnetic dipole moment is negligible as illustrated in the inset of Fig. 7. To quantify such multipole responses of the resonant cavity, we performed a multipole analysis (see Appendix B) to calculate its induced oscillating toroidal dipole moment, as well as other electric and magnetic multipole moments. In the calculations, a point dipole source is located on top of the defect rod. Figure 7 displays the relative strength and contribution to the radiation power of each multipole moment. It clearly shows that the resonance is mainly due to a toroidal dipole moment and electric quadrupole moment rather than magnetic dipole and multipole moments.

\section{DISCUSSION AND CONCLUSIONS}

This work presents an important step in realizing the localization of photons in metamaterials. In addition, by analyzing the dispersion relation of the spoof SPPs in a 2D GMA, it is shown that its polaritonic property leads to a band gap. More importantly, even without any dielectric material, we achieved a small modal volume with a high $Q$ factor. This means that strong enhancement of light-matter interaction is possible using the proposed metamaterial design. Moreover, we have found that localized spoof SPPs exhibit a significant strong toroidal dipole moment and electric quadrupole moment as well as a relatively weak electric dipole moment and higher multipole moments. This leads to the observed strong localization and a high $Q$ factor.

Besides, spoof SPPs in this work have analogies to phonon polaritons in condensed matter physics. Hence, using the concept of localizing spoof SPPs in a point defect, we could examine localized polaritons created by impurity in polar crystals. In fact, the atomic defect induced by localized polaritons were predicted theoretically long time ago [62-64]. However, they have not been observed in experiments because it is very difficult to make a single atomic defect in polar crystals. In contrast, it is easy to make a single meta-atom defect in a polaritonic metamaterial and excite localized spoof SPPs to mimic localized phonon polaritons.

The resonant cavity for spoof SPPs could provide a new architecture in the field of cavity QED or circuit QED in the microwave frequency region. In addition to those applications, although the experiments were performed in microwave region, the almost perfect conductive nature of conventional metals at $\mathrm{THz}$ frequencies would allow us to predict localization of spoof SPP modes in the $\mathrm{THz}$ region. Despite the challenging future works, such as improving coupling methods and decreasing metallic losses, we believe that our results will inspire new ideas for $\mathrm{THz}$ device applications such as resonant cavities, channel drop filters, and waveguides in on-chip platforms.

\section{ACKNOWLEDGMENTS}

This research was supported by the Basic Science Research Program through the National Research Foundation of Korea (NRF) funded by the Ministry of Education, Science and Technology (No. 2008-0062257 and NRF-2011-357-C00042) and by the APRI Research Program (Applications of Ultrashort Quantum Beam Facility) through a grant provided by the GIST in 2014. S.S.O. and O.H. acknowledge financial support from the Leverhulme Trust.

\section{APPENDIX A: DISPERSION RELATIONS FOR TE-LIKE HYBRID MODES}

Here, we derive dispersion equation of TE-like hybrid modes in a 2D GMA, using a modal expansion method under a perfect magnetic conducting wall assumption. In realistic situations, it is challenging to derive the exact dispersion equations for the TE-like hybrid mode. Therefore, we simplify this problem. First, considering guided modes propagating only in the $x$ direction, we assume that electric walls, in which the parallel components of electric fields are zero, are placed at $y= \pm a / 2$, as illustrated in Fig. 8(a). Second, if parallel components of magnetic fields are almost zero at upper boundary and the fields are well confined within the region of textured region (we neglect fringe effect), the boundary may be regarded as a magnetic wall, in which there are no parallel components of magnetic fields. At least, outside the light cone, this assumption can be justified by analyzing mode profiles obtained by FEM simulations, shown in Figs. 8(b) and $8(\mathrm{c})$. In region $\mathrm{I}(-d / 2 \leqslant y \leqslant d / 2)$, the absence of $E_{y}$ and $E_{z}$ components leads to the allowable field distributions which are written as

$$
\begin{aligned}
E_{x}^{\mathrm{I}} & =A_{0} \sin (K y) \sin \left(k_{c, m} z\right), \\
H_{y}^{\mathrm{I}} & =-\frac{A_{0} \pi}{i \omega \mu_{0} a} \sin (K y) \cos \left(k_{c, m} z\right), \\
H_{z}^{\mathrm{I}} & =-\frac{K A_{0}}{i \omega \mu_{0}} \cos (K y) \sin \left(k_{c, m} z\right),
\end{aligned}
$$

where $A_{0}$ is a constant, $K=\sqrt{{k_{0}^{2}-k_{c, m}^{2}}^{2}}$, and $k_{c, m}=(2 m-$ 1) $\pi / 2 h$ with $m$ is a positive integer $(1,2,3, \ldots)$. In region II $(d / 2 \leqslant y \leqslant a / 2)$, in the absence of the $E_{z}$ component, the 
(a)
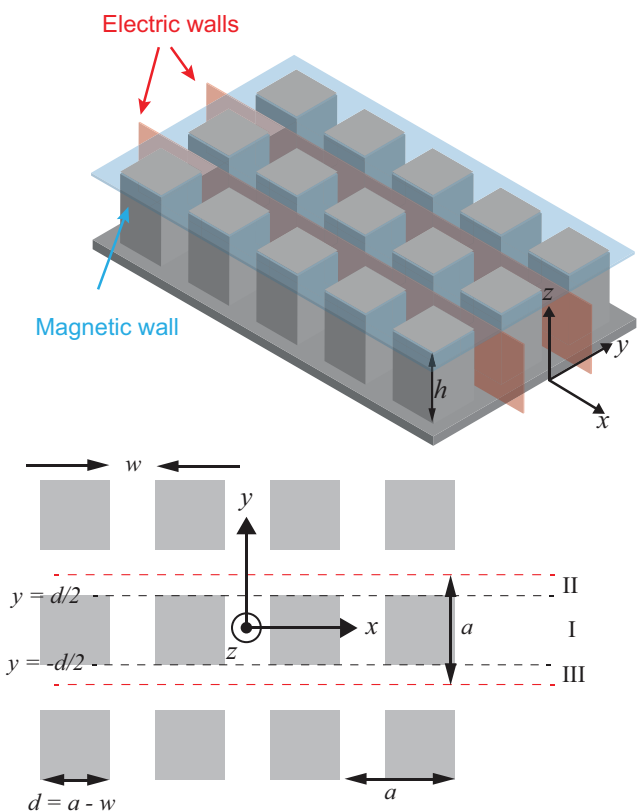

(b)

|E|

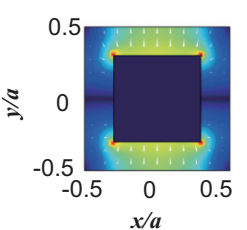

(c)
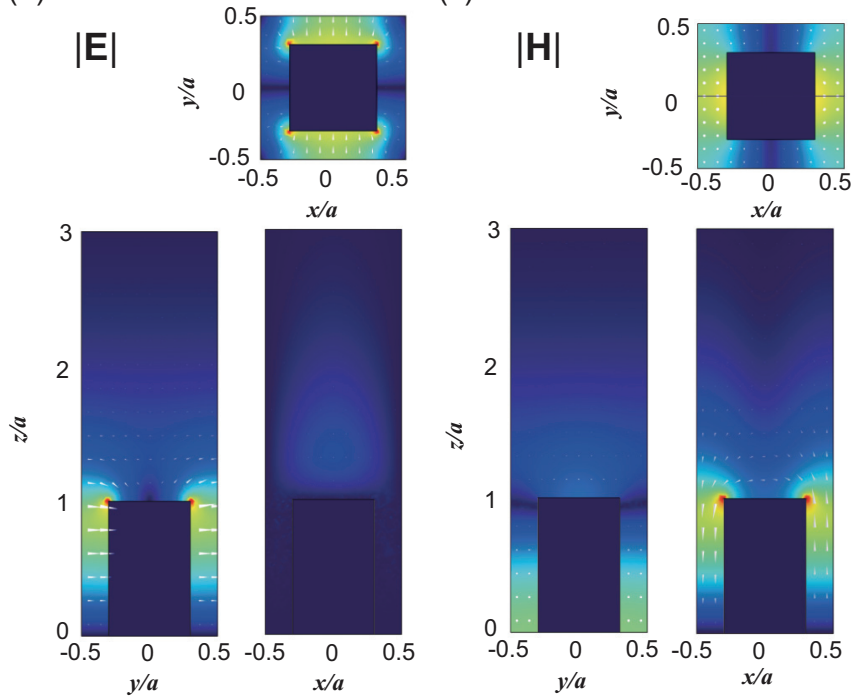

FIG. 8. (Color online) (a) Schematic diagram for deriving the approximated dispersion equation of the TE-like hybrid modes, which explains what kinds of boundary conditions can be placed. (b), (c) Calculated amplitude distributions of electric and magnetic fields for the 2D GMA with $h=a$ and $w=0.4 a$. Upper panel shows fields at $z=0.9 a$ plane, lower left one fields at $x=0$, and lower right one fields at $y=0$. The white arrows indicate the direction of fields.

allowed modes are TE-like hybrid:

$$
\begin{aligned}
E_{x}^{\mathrm{II}}= & \sum_{n=-\infty}^{\infty} B_{n} \sinh \left[\alpha_{n}\left(y-\frac{a}{2}\right)\right] \exp \left(i k_{x, n} x\right) \sin \left(k_{c, m} z\right), \\
E_{y}^{\mathrm{II}}= & \sum_{n=-\infty}^{\infty}-\frac{i k_{x, n} B_{n}}{\alpha_{n}} \cosh \left[\alpha_{n}\left(y-\frac{a}{2}\right)\right] \exp \left(i k_{x, n} x\right) \\
& \times \sin \left(k_{c, m} z\right),
\end{aligned}
$$

where $B_{n}$ is a constant, $k_{x, n}=k_{x}+2 \pi n / a$ which is related with a diffraction effect, $\alpha_{n}=\sqrt{k_{x, n}^{2}-K^{2}}$. Similarly, in region III $(-a / 2 \leqslant y \leqslant-d / 2)$,

$$
\begin{aligned}
E_{x}^{\mathrm{III}}= & \sum_{n=-\infty}^{\infty} C_{n} \sinh \left[\alpha_{n}\left(y+\frac{a}{2}\right)\right] \exp \left(i k_{x, n} x\right) \\
& \times \sin \left(k_{c, m} z\right), \\
E_{y}^{\mathrm{III}}= & \sum_{n=-\infty}^{\infty}-\frac{i k_{x, n} C_{n}}{\alpha_{n}} \cosh \left[\alpha_{n}\left(y+\frac{a}{2}\right)\right] \exp \left(i k_{x, n} x\right) \\
& \times \sin \left(k_{c, m} z\right),
\end{aligned}
$$

where $C_{n}$ is a constant. The dispersion relation of the TE-like hybrid modes are obtained by applying matching boundary conditions on tangential components of fields at the two interfaces (i.e., $y= \pm d / 2$ ). These boundary conditions yield the dispersion relation

$$
\cot \left(K \frac{d}{2}\right)=\sum_{n=-\infty}^{\infty} \frac{w K}{a \alpha_{n}} \operatorname{coth}\left(\alpha_{n} \frac{w}{2}\right) \operatorname{sinc}^{2}\left(\frac{k_{x, n} w}{2}\right)
$$

Comparison of the derived dispersion equation with numerical results for the region outside the light cone shows a good agreement, as depicted in Fig. 2, though the dispersion equation is derived under the assumption that the structure is closed by the perfect magnetic conductor at the upper boundary $(z=h)$.

\section{APPENDIX B: POWER RADIATED BY MULTIPOLES}

To understand how the multipole moments contribute to the radiations of the resonant cavity, we calculated the values of multipoles and their radiation powers using the formulas derived in Ref. [56]. For a harmonic excitation $\exp (-i \omega t)$, the following expressions represent Cartesian multipoles:

electric dipole moment:

$\mathbf{p}=\frac{1}{i \omega} \int \mathbf{j} d V$

magnetic dipole moment:

$\mathbf{m}=\frac{1}{2 c} \int(\mathbf{r} \times \mathbf{j}) d V$,

toroidal dipole moment:

$\mathbf{T}=\frac{1}{10 c} \int\left[(\mathbf{r} \cdot \mathbf{j}) \mathbf{r}-2 r^{2} \mathbf{j}\right] d V$,

electric quadrupole moment:

$Q_{\alpha \beta}=\frac{1}{i 2 \omega} \int\left[r_{\alpha} j_{\beta}+r_{\beta} j_{\alpha}-\frac{2}{3} \delta_{\alpha \beta}(\mathbf{r} \cdot \mathbf{j})\right] d V$,

magnetic quadrupole moment:

$$
M_{\alpha \beta}=\frac{1}{3 c} \int\left[(\mathbf{r} \times \mathbf{j})_{\alpha} r_{\beta}+(\mathbf{r} \times \mathbf{j})_{\beta} r_{\alpha}\right] d V,
$$

where $\mathbf{j}$ is the current density, and $c$ is the speed of light. In these formulas, we have substituted $-\nabla \cdot \mathbf{j} / i \omega$ for charge 
density $\rho$ using the continuity equation. The general expression for the total radiation power up to the order in Ref. [56] is given by

$$
I=\frac{2}{3} \frac{\omega^{4}}{c^{3}}|\mathbf{p}|^{2}+\frac{2}{3} \frac{\omega^{4}}{c^{3}}|\mathbf{m}|^{2}+\frac{4}{3} \frac{\omega^{5}}{c^{4}}|\mathbf{p} \cdot \mathbf{T}|+\frac{2}{3} \frac{\omega^{6}}{c^{5}}|\mathbf{T}|^{2}+\frac{1}{5} \frac{\omega^{6}}{c^{5}} Q_{\alpha \beta} Q_{\alpha \beta}+\frac{1}{20} \frac{\omega^{6}}{c^{5}} M_{\alpha \beta} M_{\alpha \beta},
$$

where the tensor products follow the Einstein summation convention.

[1] E. M. Purcell, Phys. Rev. 69, 681 (1946).

[2] C. Santori, D. Fattal, J. Vučković, G. S. Solomon, and Y. Yamamoto, Nature (London) 419, 594 (2002).

[3] S. M. Spillane, T. J. Kippenberg, and K. J. Vahala, Nature (London) 415, 621 (2002).

[4] J. McKeever, A. Boca, A. D. Boozer, J. R. Buck, and H. J. Kimble, Nature (London) 425, 268 (2003).

[5] C. J. Hood, T. W. Lynn, A. C. Doherty, A. S. Parkins, and H. J. Kimble, Science 287, 1447 (2000).

[6] J. M. Raimond, M. Brune, and S. Haroche, Rev. Mod. Phys. 73, 565 (2001).

[7] H. Mabuchi and A. C. Doherty, Science 298, 1372 (2002).

[8] A. Blais, R.-S. Huang, A. Wallraff, S. M. Girvin, and R. J. Schoelkopf, Phys. Rev. A 69, 062320 (2004).

[9] A. Wallraff, D. I. Schuster, A. Blais, L. Frunzio, R. S. Huang, J. Majer, S. Kumar, S. M. Girvin, and R. J. Schoelkopf, Nature (London) 431, 162 (2004).

[10] R. J. Schoelkopf and S. M. Girvin, Nature (London) 451, 664 (2008).

[11] A. Shnirman, G. Schön, and Z. Hermon, Phys. Rev. Lett. 79, 2371 (1997).

[12] Y. Nakamura, Y. A. Pashkin, and J. S. Tsai, Nature (London) 398, 786 (1999).

[13] V. Bouchiat, D. Vion, P. Jouez, D. Esteve, and M. H. Devoret, Phys. Scr. T 76, 165 (1998).

[14] C. Hermann and O. Hess, J. Opt. Soc. Am. B 19, 3013 (2002).

[15] S. Noda, A. Chutinan, and M. Imada, Nature (London) 407, 608 (2000).

[16] Y. Akahane, T. Asano, B.-S. Song, and S. Noda, Nature (London) 425, 944 (2003).

[17] J. S. Foresi, P. R. Villeneuve, J. Ferrera, E. R. Thoen, G. Steinmeyer, S. Fan, J. D. Joannopoulos, L. C. Kimerling, H. I. Smith, and E. P. Ippen, Nature (London) 390, 143 (1997).

[18] H. Raether, in Surface Plasmons (Springer-Verlag, Berlin, 1988), Chap. 2.

[19] M. Moskovits, Rev. Mod. Phys. 57, 783 (1985).

[20] L. Rogobete, F. Kaminski, M. Agio, and V. Sandoghdar, Opt. Lett. 32, 1623 (2007).

[21] A. Mohammadi, V. Sandoghdar, and M. Agio, New J. Phys. 10, 105015 (2008).

[22] M. Kuttge, F. J. García de Abajo, and A. Polman, Nano Lett. 10, 1537 (2010).

[23] J. Aizpurua, P. Hanarp, D. S. Sutherland, M. Käll, G. W. Bryant, and F. J. García de Abajo, Phys. Rev. Lett. 90, 057401 (2003).

[24] E. J. A. Kroekenstoel, E. Verhagen, R. J. Walters, L. Kuipers, and A. Polman, Appl. Phys. Lett. 95, 263106 (2009).

[25] I. Bulu, T. Babinec, B. Hausmann, J. T. Choy, and M. Loncar, Opt. Express 19, 5268 (2011).

[26] I. S. Maksymov, M. Besbes, J. P. Hugonin, J. Yang, A. Beveratos, I. Sagnes, I. Robert-Philip, and P. Lalanne, Phys. Rev. Lett. 105, 180502 (2010).

[27] C. Genet and T. W. Ebbesen, Nature (London) 445, 39 (2007).
[28] T. Pickering, J. M. Hamm, F. A. Page, S. Wuestner, and O. Hess, Nat. Commun. 5, 4972 (2014).

[29] J. B. Pendry, L. Martín-Moreno, and F. J. García-Vidal, Science 305, 847 (2004).

[30] F. J. García-Vidal, L. Martín-Moreno, and J. B. Pendry, J. Opt. A: Pure Appl. Opt. 7, S97 (2005).

[31] A. P. Hibbins, B. R. Evans, and J. R. Sambles, Science 308, 670 (2005).

[32] F. J. García de Abajo and J. J. Sáenz, Phys. Rev. Lett. 95, 233901 (2005).

[33] S. A. Maier, S. R. Andrews, L. Martín-Moreno, and F. J. García-Vidal, Phys. Rev. Lett. 97, 176805 (2006).

[34] C. R. Williams, S. R. Andrews, S. A. Maier, A. I. FernándezDomínguez, L. Martín-Moreno, and F. J. García-Vidal, Nat. Photonics 2, 175 (2008).

[35] S. S. Oh, S.-G. Lee, J.-E. Kim, and H. Y. Park, Opt. Express 15, 1205 (2007).

[36] E. Moreno, S. G. Rodrigo, S. I. Bozhevolnyi, L. Martín-Moreno, and F. J. García-Vidal, Phys. Rev. Lett. 100, 023901 (2008).

[37] W. Zhao, O. M. Eldaiki, R. Yang, and Z. Lu, Opt. Express 18, 21498 (2010).

[38] S.-H. Kim, T.-T. Kim, S. S. Oh, J.-E. Kim, H. Y. Park, and C.-S. Kee, Phys. Rev. B 83, 165109 (2011).

[39] X. Shen and T. J. Cui, Appl. Phys. Lett. 102, 211909 (2013).

[40] K.-J. Kim, J.-E. Kim, H. Y. Park, Y.-H. Lee, S.-H. Kim, S.-G. Lee, and C.-S. Kee, Opt. Express 22, 4050 (2014).

[41] D. Martín-Cano, O. Quevedo-Teruel, E. Moreno, L. MartínMoreno, and F. J. García-Vidal, Opt. Lett. 36, 4635 (2011).

[42] Y. G. Ma, L. Lan, S. M. Zhong, and C. K. Ong, Opt. Express 19, 21189 (2011).

[43] A. Pors, E. Moreno, L. Martín-Moreno, J. B. Pendry, and F. J. García-Vidal, Phys. Rev. Lett. 108, 223905 (2012).

[44] A. I. Fernández-Domínguez, E. Moreno, L. Martín-Moreno, and F. J. García-Vidal, Opt. Lett. 34, 2063 (2009).

[45] D. Martín-Cano, M. L. Nesterov, A. I. Fernández-Domínguez, F. J. García-Vidal, L. Martín-Moreno, and E. Moreno, Opt. Express 18, 754 (2010).

[46] Z. Gao, L. Shen, J.-J. Wu, T.-J. Yang, and X. Zheng, Opt. Commun. 285, 2076 (2012).

[47] S. J. Berry, T. Campbell, A. P. Hibbins, and J. R. Sambles, Appl. Phys. Lett. 100, 101107 (2012).

[48] V. Savinov, V. A. Fedotov, and N. I. Zheludev, Phys. Rev. B 89, 205112 (2014).

[49] Y. Fan, Z. Wei, H. Li, H. Chen, and C. M. Soukoulis, Phys. Rev. B 87, 115417 (2013).

[50] Z.-G. Dong, J. Zhu, X. Yin, J. Li, C. Lu, and X. Zhang, Phys. Rev. B 87, 245429 (2013).

[51] Z.-G. Dong, P. Ni, J. Zhu, X. Yin, and X. Zhang, Opt. Express 20, 13065 (2012).

[52] Y.-W. Huang, W. T. Chen, P. C. Wu, V. Fedotov, V. Savinov, Y.Z. Ho, Y.-F. Chau, N. I. Zheludev, and D. P. Tsai, Opt. Express 20, 1760 (2012). 
[53] Z.-G. Dong, J. Zhu, J. Rho, J.-Q. Li, C. Lu, X. Yin, and X. Zhang, Appl. Phys. Lett. 101, 144105 (2012).

[54] V. A. Fedotov, A. V. Rogacheva, V. Savinov, D. P. Tsai, and N. I. Zheludev, Sci. Rep. 3, 2967 (2013).

[55] V. M. Dubovik and V. V. Tugushev, Phys. Rep. 187, 145 (1990).

[56] E. E. Radescu and G. Vaman, Phys. Rev. E 65, 046609 (2002).

[57] T. Kaelberer, V. A. Fedotov, N. Papasimakis, D. P. Tsai, and N. I. Zheludev, Science 330, 1510 (2010).

[58] D. M. Pozar, Microwave Engineering, 3rd ed. (Wiley, New York, 2004).
[59] C. Kittel, Introduction to Solid State Physics, 7th ed. (Wiley, New York, 1996).

[60] J. D. Joannopoulos, S. G. Johnson, J. N. Winn, and R. D. Meade, Photonic Crystals: Molding the Flow of Light, 2nd ed. (Princeton University Press, New Jersey, 2008).

[61] J. T. Robinson, C. Manolatou, L. Chen, and M. Lipson, Phys. Rev. Lett. 95, 143901 (2005).

[62] G. F. Koster and J. C. Slater, Phys. Rev. 95, 1167 (1954).

[63] L. I. Deych and A. A. Lisyansky, Phys. Lett. A 240, 329 (1998).

[64] A. A. Maradudin and J. Oitmaa, Solid State Commun. 7, 1143 (1969). 